\title{
AUGMENTED MUSCLE ACTIVATION IN REACHING MOVEMENTS AFTER STROKE
}

original paper

( ) University School of Physical Education in Wroclaw

DOI: https://doi.org/10.5114/hm.2020.91344

\section{PAULA CINTIA DOS SANTOS VIEIRA, RAFAEL ALMEIDA OLIVEIRA, LUCIANE MARTINHO FERNANDES, KARINA PEREIRA, LUCIANE APARECIDA PASCUCCI SANDE DE SOUZA}

Federal University of Triangulo Mineiro, Uberaba, Brazil

\begin{abstract}
Purpose. Post-stroke hemiparesis can cause motor deficits that affect the completion of functional activities, such as reaching. The study aim was to quantitatively compare the changes in muscle activation between hemiparetic individuals during the subacute and chronic phases of recovery and healthy individuals in a reaching task.

Methods. The electromyographic activity was recorded of the upper trapezius (UT), biceps brachii (BB), triceps brachii (TB), flexor carpi ulnaris (FCU), and extensor carpi radialis (ECR) muscles in 10 subacute individuals (group 1), 10 chronic individuals (group 2), and 10 healthy individuals (group 3). The normalized root mean square (nRMS) was calculated, followed by a qualitative index based on maximum activation, and the occurrence of co-contraction was analysed.

Results. In the nRMS analysis, group 1 presented significantly higher activation than group 3 for BB, TB, and ECR; group 2 showed significantly higher activation than group 3 for BB and FCU. In the qualitative analysis, group 1 exhibited greater activity for UT, group 2 for ECR, and group 3 for UT. There was no statistically significant difference in the co-contraction analysis between the antagonist muscle pairs of BB/TB and FCU/ECR.
\end{abstract}

Conclusions. Muscle activation was greater in the post-stroke groups in comparison with healthy individuals.

Key words: stroke, hemiparesis, electromyography

\section{Introduction}

Stroke is characterized as a neurological deficit attributed to an acute focal injury of the central nervous system by a vascular cause [1]. Each year, 795,000 people suffer from a new or recurring [2] stroke, and only $40 \%$ of survivors recover completely [3], which makes stroke one of the principal causes of disability in the world [4].

Hemiparesis is the most common outcome in poststroke individuals and it generally refers to the heterogeneous condition comprised of muscle weakness, changes in motor control, and spasticity [5]. Among these, muscle weakness was the strongest and therefore is considered the factor most responsible for limitations in daily activities [6]; it is assigned to inadequate recruitment of motor units, resulting in an inability to generate sufficient force [7].
Thus, changes can be observed in muscle activation patterns, such as loss of selective activation of muscle groups necessary to execute specific motor tasks and incorrect proportion of muscle pairs activation as cocontraction of antagonist muscles [8]. Muscle co-contraction can be related to reduced inhibition [7] or lack of muscle activation [8]. According to Gowland et al. [7], co-contraction is related to the fact that the inhibiting action would be negatively impacted on the paretic side. Still, Wagner et al. [8] affirm that co-contraction can be associated with an impairment in the activation of the agonist muscle, and not with excessive activation of the antagonist muscle.

McCrea et al. [9] describe a saturation in muscle activation in post-stroke patients and affirm that it would also be related to muscle weakness, requiring additional muscle activation during the execution of movements. In the same way, Trombly [10] observed

Correspondence address: Luciane Aparecida Pascucci Sande de Souza, Federal University of Triangulo Mineiro, Frei Paulino Avenue, 30, Abadia, Zip code 38025-180, Uberaba, Minas Gerais, Brazil, e-mail: lucianesande@gmail.com

Received: November 12, 2018

Accepted for publication: October 16, 2019

Citation: dos Santos Vieira PC, Oliveira RA, Fernandes LM, Pereira K, de Souza LAPS. Augmented muscle activation in reaching movements after stroke. Hum Mov. 2020;21(3):39-46; doi: https://doi.org/10.5114/hm.2020.91344. 
that these individuals used a significantly higher percentage of available activity in the motor units of the paretic limb.

In this context, loss of some functional abilities can be observed; among them is reaching [11]. The ability to achieve adequate reach is a fundamental component of daily activities [12] and, considering that this task requires precise planning [13, 14], damage to the central nervous system can result in reduced capacity to activate and modulate the muscles involved in its execution [8]. Therefore, the individual will often compensate [15] in the execution of motor tasks, e.g. excessive activation of the upper trapezius (UT) muscle can be regarded a compensating strategy in movements of raising the upper limb [16].

It can be assumed that, in accordance with the poststroke recovery process, the individuals present differences with respect to muscle activation. Thus, the objective of this study was to quantitatively compare the changes in muscle activation during the execution of a reaching task between hemiparetic individuals in the subacute and chronic stages of recovery and individuals with no history of stroke.

Our initial hypotheses were: (1) when compared with individuals without history of stroke, post-stroke patients will show greater use of the capacity to activate each muscle during the performance of a task, this being more evident among the subacute group than in the chronic group; (2) there will be greater activation of UT in the subacute group when compared with the other groups, as a means of compensating for the reaching movement; and (3) the proportion of activation in the antagonist pairs of biceps brachii (BB)/triceps brachii (TB) and flexor carpi ulnaris (FCU)/extensor carpi radialis (ECR) among post-stroke individuals will predominate with the two antagonistic muscles activating at the same proportion through the entire task duration.

\section{Material and methods}

A total of 24 stroke survivors from the Neurology Department of Applied Physiotherapy, as well as 10 individuals with no history of stroke met the inclusion criteria. The inclusion criteria for the post-stroke individuals were: hemiparesis lasting for more than 3 months and continued physician visits, age range of 45-75 years, no musculoskeletal system diseases, no cognitive deficit, and having signed the informed consent form after having read it and understood it completely. Overall, 4 hemiparetic individuals were not able to complete the reaching task. Therefore, 30 sub- jects were assessed in total: 10 in group 1 (subacute, less than 6 months post-stroke [17]), 10 in group 2 (chronic, more than 6 months post-stroke), and 10 in group 3 (individuals with no history of stroke and no musculoskeletal disorders, which, in this case, we considered the healthy group). The dominant arm in group 3 participants was tested in this research because, according to McCrea et al. [9], the dominant arm in healthy individuals displays more muscle activation than the non-dominant one. The status of paresis severity was assessed by the Fugl-Meyer score of the upper extremity, where all the stroke survivors should be able to reach with full elbow extension. We also used the Barthel index to evaluate the independence level of the stroke survivors.

The collections were performed in the Biomechanical and Motor Control Laboratory of the Federal University of Triangulo Mineiro for a period of 12 months.

To perform the reaching task, the individuals were placed in a sitting position with hips, knees, and ankles flexed at $90^{\circ}$, shoulders in a neutral position, and elbows flexed at $75-90^{\circ}$ [18]. The trunk was stabilized with a Velcro belt to avoid compensatory strategy. The participants were asked to reach for a target placed at the height of the xiphoid process, at a distance equivalent to the functional length of the arm when the individual raised it to $90^{\circ}$ in a flexed position [19] on the sagittal plane [20] (Figure 1). Necessary measures were put in place to minimize any potential positional discomfort, including rest times between the trials and the use of a pillow to rest the arm on.

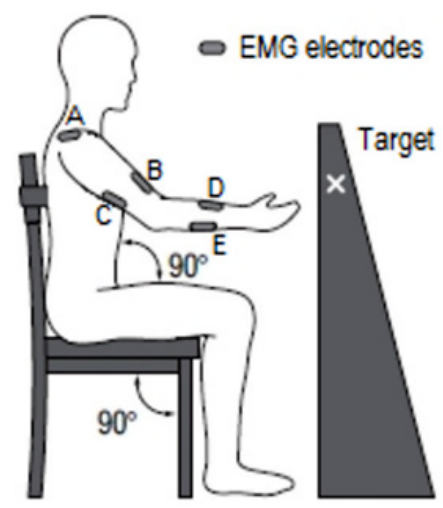

Figure 1. Positioning of the individuals, the target to be reached, and the electrodes on the assessed muscles:

(A) upper trapezius, (B) biceps brachii, (C) triceps brachii,

(D) flexor carpi ulnaris, (E) extensor carpi radialis

For the electromyographic evaluation, disposable electrodes were placed on each muscle pair to be assessed. Prior to placing the electrodes, the area was trichotomized, cleaned with $70 \%$ alcohol, and exfoli- 
ated with a fine abrasive in order to reduce the skin's impedance [21].

The placement of the electrodes followed the guidelines laid out in the SENIAM (Surface Electromyography for the Non-Invasive Assessment of Muscles) [21] protocol for UT, BB, and TB. For FCU and ECR, the study by Lima et al. [22] was followed, which had also been applied in other research [18, 23]. Three reaching movements were carried out. During the data collection, all electronic equipment within the room and in nearby rooms were turned off to avoid interferences.

The electromyographic assessment of the muscles was recorded by an EMG System do Brasil ${ }^{\oplus}$ electromyograph (São José dos Campos, Brazil), with a bandpass of $20-500 \mathrm{~Hz}$, common-mode rejection greater than $-120 \mathrm{~dB}$, input impedance greater than $10 \mathrm{M} \Omega$, and gains of $\times 100$ in the signal conditioner and $\times 20$ in the bipolar electrode preamplifier (total gain of 2000). WinDaq software (DATAQ Instruments, Akron, USA) was used for the analysis of data acquired at a sampling frequency of $1 \mathrm{kHz}$ for each channel.

The electromyographic signal was collected at $1000 \mathrm{~Hz}$, and later filtered. The values for the signal amplitude were represented by the root mean square (RMS) over the entire duration of the task and then normalized by the ratio between the RMS value and the maximum value obtained during the maximum voluntary isometric contraction (MVIC) of each muscle, for the 3 repetitions of the reaching task (equation 1a). Therefore, the average values obtained were considered. In order to represent the muscle activation of the reaching task in proportion to the maximum muscle activation, the normalized RMS (nRMS) was multiplied by 100 and expressed in the activation percentage value.

After calculating the nRMS, a qualitative index was created on the basis of the maximum value of the electromyographic activation between the 5 muscles, representing how much each muscle, to its maximum capacity, contributed to the successful execution of the task. The muscle with greatest activation was considered at $100 \%$ and the others as a proportional percentage of this index.

In order to assess the proportion of activation between the antagonist pairs (BB and TB, and FCU and ECR), we modified the formula described by Hammond et al. [24] (equations 1b and 1c), which considers the proportion of antagonistic activity in relation to total electromyographic activity, as well as agonistic and antagonistic activities as an indicator for coactivation. As other authors [23, 25], we also used the nRMS values of each muscle over the entire task time to calculate the proportion of the muscle activation in each antagonist pair. With this method, it is possible to analyse the prevalence of muscle activation in the antagonistic pairs analysed. The method is important for clinical applicability because the view of the predominance of certain muscle activation enables the development of strategies that focus on a targeted treatment of the specific muscles with higher and lower neuromuscular activation.

$$
\begin{aligned}
& n R M S=\frac{R M S[A U]}{M V C[\mu V]} \\
& \text { (b) } \\
& \text { (a)-contraction } B B / T B=\frac{E M G B B}{E M G B B+E M G T B} \times 100
\end{aligned}
$$

Co-contraction ECR/FCU $=\frac{E M G E C R}{E M G E C R+E M G F C U} \times 100$

Equation 1. (a) nRMS - normalized data for electromyographic activation, RMS - root mean square, MVC - maximum voluntary contraction value, $\mathrm{AU}$ - arbitrary unit, $\mu \mathrm{V}$ - microvolt; (b) $\mathrm{BB}$ - biceps brachii muscle, TB - triceps brachii muscle, EMG - electromyographic activity; (c) ECR - extensor carpi radialis, FCU - flexor carpi ulnaris, EMG - electromyographic activity

The data were processed by using Excel and KaleidaGraph 4.0 (Synergy Software, Reading, USA). Statistica software was used for the statistical analysis. In order to ensure unbiased results, the data were analysed by professionals who did not participate in the clinical evaluation and the electromyographic examination of the individuals. Data normality was verified by the Shapiro-Wilk test. The nRMS data did not represent normal distribution and the non-parametric Mann-Whitney U test was used for intergroup evaluation. As the co-contraction data presented normal distribution, they were subjected to the one-way ANOVA test and the post-hoc Tukey test. The nRMS values were considered as dependent variables and the other parameters (group and muscle) as independent variables. For the co-contraction analysis, the independent variable was the group, and the dependent variable was the value of the co-contraction of the analysed pairs.

\section{Ethical approval}

The research related to human use has complied with all the relevant national regulations and institu- 


\section{HUMAN MOVEMENT}

P. Vieira et al., Post-stroke reaching movements: augmented muscle activation

tional policies, has followed the tenets of the Declaration of Helsinki, and has been approved by the Research Ethics Committee at the Federal University of Triangulo Mineiro, under protocol No. 1647.

\section{Informed consent}

Informed consent has been obtained from all individuals included in this study.
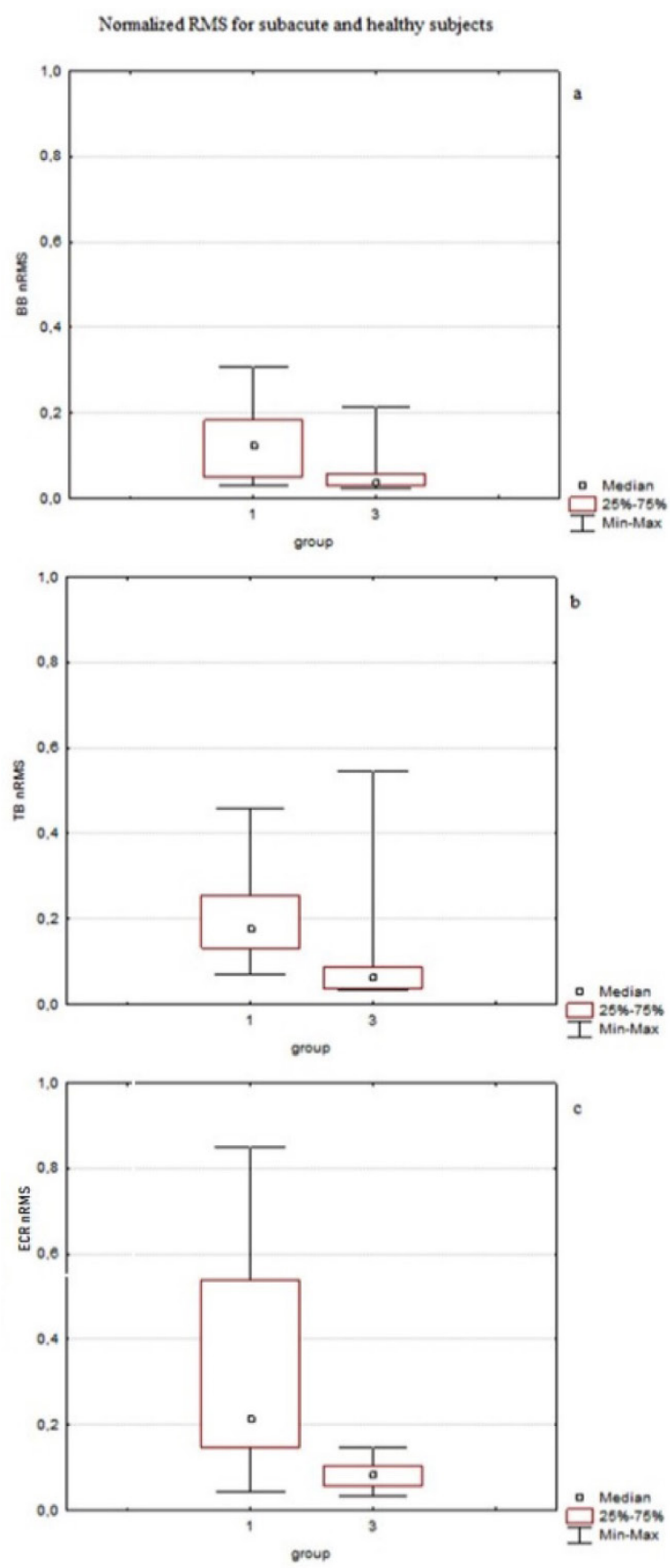

\section{Results}

The profile of the individuals is presented in Table 1. The subacute post-stroke group presented $60.82( \pm 4.92)$ Fugl-Meyer score and the value for the chronic poststroke group was $59.50( \pm 7.12)$. All patients were classified as mild to moderate functional independence in accordance with the index of Barthel. The subacute post-stroke group presented significantly higher
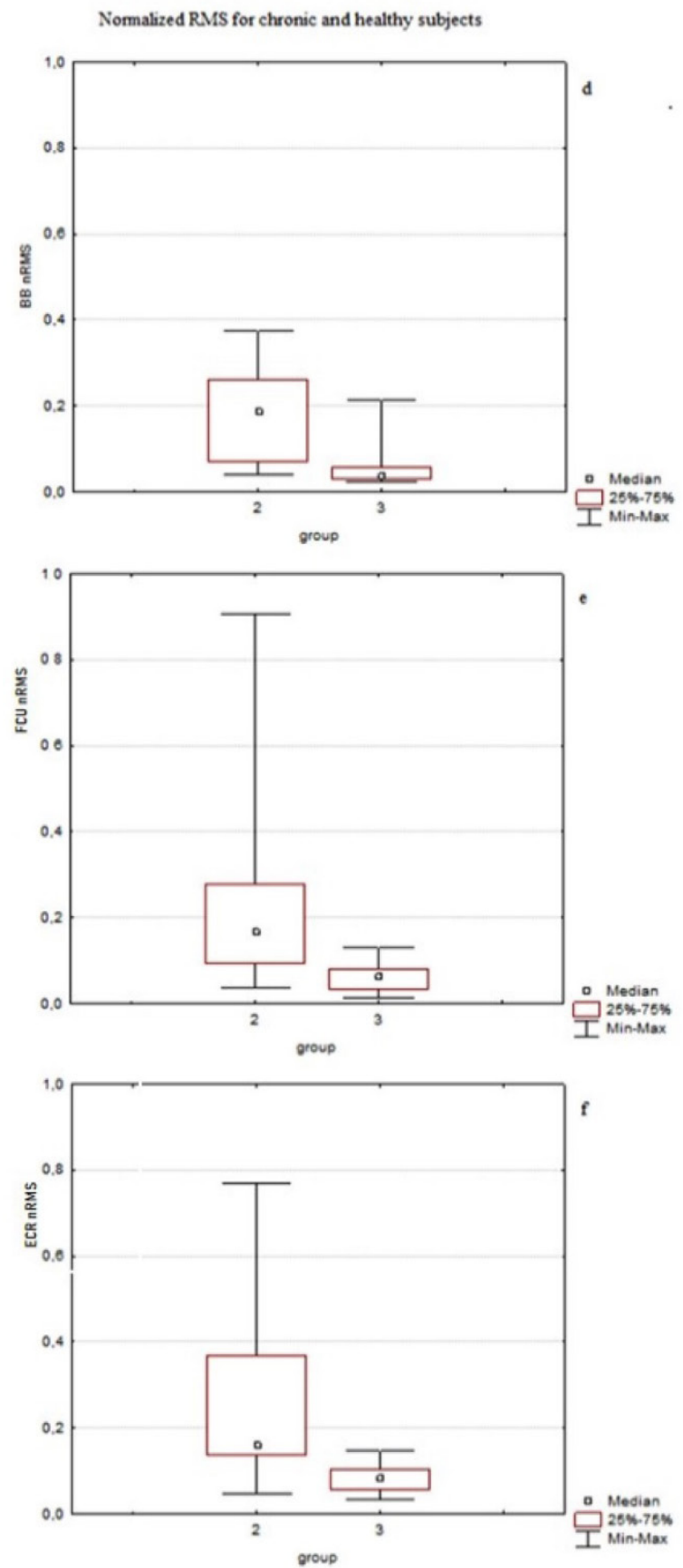

RMS - root mean square, nRMS - normalized RMS, BB - biceps brachii, TB - triceps brachii, ECR - extensor carpi radialis,

FCU - flexor carpi ulnaris; groups: 1 - subacute, 2 - chronic, 3 - healthy

Figure 2. Comparison of nRMS values for each muscle category: $(\mathrm{a}-\mathrm{c})$ the paretic side in group 1 and the dominant side in group 3, (d-f) the paretic side in group 2 and the dominant side in group 3 
Table 1. Profile of the study participants

\begin{tabular}{lccc}
\hline Variable & $\begin{array}{c}\text { Subacute group } \\
(n=10)\end{array}$ & $\begin{array}{c}\text { Chronic group } \\
(n=10)\end{array}$ & $\begin{array}{c}\text { Healthy group } \\
(n=10)\end{array}$ \\
\hline Age (years), average $(S D)$ & $59.18( \pm 11.92)$ & $59( \pm 12.37)$ & $54.7( \pm 12.36)$ \\
Sex, men/women & $8 / 2$ & $6 / 4$ & $4 / 6$ \\
Hemiparetic side, right/left & $6 / 4$ & $4 / 6$ & - \\
Time since stroke (months), average (SD) & $3.36( \pm 0.81)$ & $10.2( \pm 1.55)$ & - \\
Tonus, modified Ashworth scale (0-4) & $1(100 \%)$ & $1(100 \%)$ & - \\
Fugl-Meyer score & $60.82( \pm 4.92)$ & $59.50( \pm 7.12)$ & - \\
Barthel index, mild/moderate level of function & $8 / 2$ & $8 / 2$ & - \\
\hline
\end{tabular}

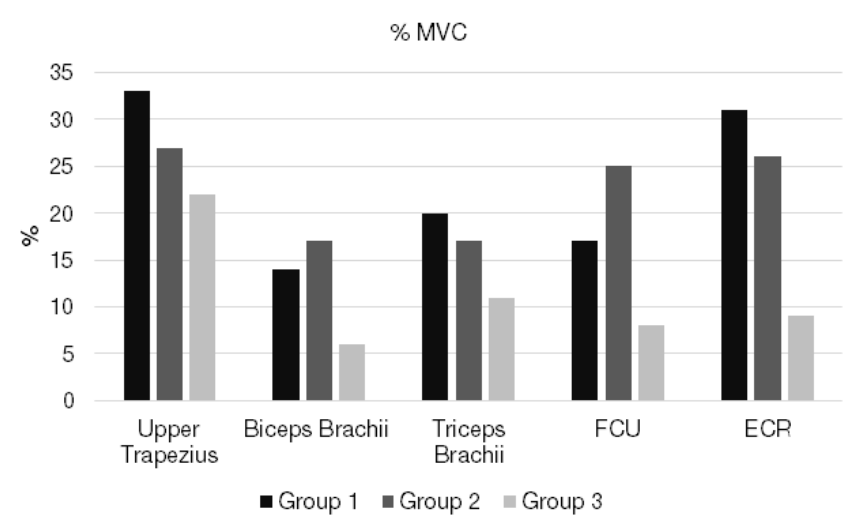

$M V C$ - maximum voluntary contraction, FCU - flexor carpi ulnaris, ECR - extensor carpi radialis

Figure 3. Relative percentage of muscular activation on the basis of maximum voluntary contraction

activation than the healthy group for $\mathrm{BB}(\mathrm{U}=21.0$, $p=0.02), \mathrm{TB}(\mathrm{U}=20.0, p=0.02)$, and $\mathrm{ECR}(\mathrm{U}=12.0$, $p=0.004$ ) (Figure 2a, b, c). The chronic post-stroke group presented significantly higher activation than group 3 for $\mathrm{BB}(\mathrm{U}=13.0, p=0.005)$ and $\mathrm{FCU}(\mathrm{U}=14.0$, $p=0.006)$; the activation was also higher, although not significantly, for ECR ( $\mathrm{U}=15.0, p=0.008)$ (Figure $2 \mathrm{~d}, \mathrm{e}, \mathrm{f})$. Significant differences were not found when comparing groups 1 and 3 for UT and FCU, or between groups 2 and 3 for UT and TB.

Figure 3 represents the muscle activation during reaching in proportion to the maximum activation obtained in the MVIC test for each muscle. The nRMS values are expressed as a percentage for groups 1,2, and 3. Group 1 presents higher activation values for all muscles except BB and ECR, indicating that, in comparison with the chronic and healthy groups, the individuals in the subacute post-stroke phase needed greater muscle activation to complete the functional reaching task.

During a qualitative intergroup analysis, the values of the rates for groups 1,2, and 3 were considered separately, and activities were compared between the paretic side in groups 1 and 2, and the dominant side in group 3. For group 1, UT activity was predominant, which represents $100 \%$ in this analysis, followed by the activity of TB (67.60\%), ECR (66.25\%), BB (41.73\%), and FCU (41.55\%). In group 2, the predominant activity was that of ECR (100\%), followed by FCU (83.01\%), UT (80.81\%), BB (60.21\%), and TB (54\%). In group 3, the greatest activity was for UT (100\%), followed by TB (73.71\%), ECR (34.87\%), BB (27.66\%), and FCU (26.12\%).

In the analysis of the proportion of activation between the antagonist pairs, there was no significant statistical difference between the groups for the $\mathrm{BB} / \mathrm{TB}$ pairs $(\mathrm{F}(2.27)=1.113, p=0.343)$ or $\mathrm{FCU} / \mathrm{ECR}(\mathrm{F}(2.27)$ $=0.990, p=0.385)$.

\section{Discussion}

The objective of this study was to quantitatively compare muscle activation between hemiparetic individuals in the subacute and chronic phases of recovery and participants without stroke history, keeping in mind that changes in this activation, related to hemiparesis, could interfere in the implementation of the reaching task. On the basis of the results, we can consider that this objective was achieved, as $2 / 3$ of our hypotheses were confirmed.

We first hypothesized that for post-stroke individuals, we would find greater usage of activation capacity for each muscle, with the highest evidence in group 1. The substrate for this hypothesis is that after each injury occurs, the individuals will not have control over the modulation of muscle activation in the performance of the task; therefore, this deficit would diminish after a certain time post-stroke [8].

The results of the nRMS analysis reveal that the subjects in the post-stroke groups (subacute and chronic), while performing the functional reaching movement, present higher activation values for all muscles analysed, except UT, when compared with the healthy group. Since the RMS data of this study were 
normalized by using the MVIC test values, our results show that, on the basis of maximum muscle capacity, the individuals in the post-stroke groups needed greater muscle activation to execute the same functional reaching movement as those without a history of stroke.

Other studies have also reported greater muscle usage rates during the reaching movement among poststroke participants [8, 9]. Applying the MVIC values in the analysis of activation percentage of the deltoid, TB, BB, and brachioradialis muscles, McCrea et al. [9] observed that post-stroke patients used greater muscle activity to correctly accomplish functional reach. Also, comparing the highest activation value during reaching movements with the highest value during the manual MVIC test for the deltoid, BB, TB, ECR, and flexor carpi radialis muscles, Wagner et al. [8] found that individuals in the acute and subacute post-stroke phases presented a significant increase in the use of maximum muscle capacity during the reaching movement in comparison with those without a history of stroke.

After the MVIC analysis, followed by attempts of the individuals to reach $20 \%, 40 \%, 60 \%, 80 \%$, and $100 \%$ of the MVIC value, Chang et al. [26] observed that post-stroke patients had difficulty in maintaining constant intensity during MVIC of paretic muscles, which resulted in an increase in force variability. The authors attributed this result to a reduction in the recruitment and modulation capacity of motor units, including fluctuation in the release rate of motor units and synchronization during the production of force.

Furthermore, analysing the relationship between electromyographic activity and the isometric force generated by the bilateral elbow flexors of hemiparetic individuals over a period of more than 4 months, Tang and Rymer [27] observed that despite the level of isometric force being almost identical in both sides under study, the rectified electromyographic activity was higher on the paretic side. As this was attributed to a low amount of force generated by the muscle fibres, higher recruitment of the motor units was necessary to produce an adequate level of force. The authors ascribed this result to the fact that the release rate of an individual motor unit was reduced in the paretic state.

In this project, there were no differences when comparing UT with FCU between groups 1 and 3 , and UT with TB between groups 2 and 3. Wagner et al. [8] also report that when comparing individuals in the acute and subacute phases of recovery, some muscles in some participants were found to be more active in the first phase but not in the second, and vice versa. This demonstrates that the activation pattern varied between individuals during post-stroke recovery.

The second hypothesis referred to a greater activation of UT in the subacute group. This hypothesis was supported by the knowledge that owing to the deficits presented by these individuals, their natural reaction would be to use available motor strategies as a means of compensation [15, 18].

In the analysis of the indices of muscle activation, the greater activation of UT in the healthy group could be explained by the fact that this muscle plays an important role in scapular stabilization during upper limb movements [28]. In the subacute group, UT was also very active, which confirms the proposed hypothesis; the individual shows the paretic upper limb a little more pendant owing to the muscle weakness, which would require a greater force of the UT for stabilizing the humeral head in the glenoid cavity.

In the chronic group, the predominant activity of ECR, followed by FCU, indicates greater stabilization for wrist control. According to Twitchell [29], the recovery process goes from the proximal to the distal. Thus, in individuals in the chronic phase, improvement in motor control, which is illustrated in our study by greater wrist control, would be more evident than in those in the subacute phase, who presented distinctive activation of these two muscles.

Also in line with these findings, it is interesting to note that many stroke survivors experience reasonable motor recovery in the proximal segment, with limited distal recovery [25]. Boyd et al. [30] cite that many cases of motor learning and performance improvement are observed in post-stroke individuals in the chronic phase of recovery, indicating a functional organization of the motor system that is modified by use.

The third hypothesis of our study comprised the proportion of activation between the antagonist pairs of $\mathrm{BB} / \mathrm{TB}$ and FCU/ECR in post-stroke patients when compared with individuals who had no history of stroke. This hypothesis was based on the fact that abnormal activation of agonist and antagonist muscles is a post-stroke clinical phenomenon and could be a crucial component of motor deficiency [7].

The results of the proportion of activation analysis can be related to a previous study by Trombly [10], which reported that after analysing the task of reaching in hemiparetic individuals, no differences were found in muscular co-activation between BB and TB. The author attributed this result to the differences in activation of each individual muscle. Similarly, our study did not reveal any difference between the proportions of the muscle pairs. 
Even with our analysis being performed over the entire time of the muscle pairs activation, Wagner et al. [8], who analysed the co-activation between the muscle onset and offset, also did not find statistically significant differences in the agonist and antagonist activation in post-stroke survivors. Therefore, once in the entire task duration or between the muscle onset and offset, lack of statistical significance indicates that the proportion of activation between the pairs is the same. It is an interesting analysis because it suggests that the treatment for both subacute and chronic post-stroke survivors must focus on both agonist and antagonist muscles in order to promote the correct proportion of muscle activation and relaxation.

Also, we can say that the deficit in the performance of the paretic member occurs as a result of a deficiency in the agonist muscle activation, and not because of excessive activation of the antagonist muscle [8], still present in chronic post-stroke survivors.

The novelty of the present study is the finding related to chronic individuals, who presented with more activation of distal muscles compared with the subacute and healthy groups. The use of the index analysis allowed to visualize the relationship between the 5 muscles involved in the task which reflects the recovery process. In the chronic group, the distal muscles of the upper limb were more activated, indicating acquisition of motor control, whilst in the acute group, UT was the more activated muscle, pointing at compensation in the task execution. In the healthy group, the most active muscle was UT, possibly reflecting the function of this muscle in the scapular stabilization during humeral elevation. This could help in the rehabilitation process in the sense that it is important to know how the recovering process occurs in the transition from the subacute phase to the chronic phase after stroke.

The differences found in muscular activation amongst hemiparetic individuals and those without stroke history show that since the first group needs more activation of total muscular capacity to implement the reaching task throughout the day, at least the muscles assessed in this study are prone to showing greater muscular fatigue. Therefore, clinical practice should be attentive to training the entire set of upper limb, strengthening the weak muscles, relaxing the muscles that are prone to fatigue, and providing functional training for primary daily activities in order to encourage relearning of the motor task.

In order to establish a connection between the changes in muscular activation and the compensations for movement or even the dynamic process of motor re- covery of the upper limbs in hemiparetic individuals, new studies are needed involving longitudinal methodologies and greater numbers of participants.

\section{Conclusions}

The results of this study show that in post-stroke individuals, there is greater muscular activation of the paretic limb in comparison with the dominant limb in those without a history of stroke. Post-stroke compensatory measures predominantly involve UT activation in patients in the subacute phase, and ECR activation in those in the chronic phase, which indicates compensations and greater motor control, respectively. Furthermore, we did not observe the presence of co-contraction between the antagonist pairs of the elbow and wrist, which seems to be associated with the lack of activation of the agonist muscle in the movement, and not with excessive antagonist activation.

\section{Disclosure statement}

No author has any financial interest or received any financial benefit from this research.

\section{Conflict of interest}

The authors state no conflict of interest.

\section{References}

1. Sacco RL, Kasner SE, Broderick JP, Caplan LR, Connors JJ (Buddy), Culebras A, et al. An updated definition of stroke for the $21^{\text {st }}$ century: a statement for healthcare professionals from the American Heart Association/American Stroke Association. Stroke. 2013;44(7): 2064-2089; doi: 10.1161/STR.0b013e318296aeca.

2. Mozaffarian D, Benjamin EJ, Go AS, Arnett DK, Blaha MJ, Cushman M, et al. Heart disease and stroke statistics - 2015 update: a report from the American Heart Association. Circulation. 2015;131(4):e29-e322; doi: 10.1161/CIR.0000000000000152.

3. Hankey GJ, Jamrozik K, Broadhurst RJ, Forbes S, Anderson CS. Long-term disability after first-ever stroke and related prognostic factors in the Perth Community Stroke Study. Stroke. 2002;33(4):1034-1040; doi: 10.1161/01.str.0000012515.66889.24.

4. Hong K-S, Saver JL. Quantifying the value of stroke disability outcomes: WHO global burden of disease project disability weights for each level of the modified Rankin scale. Stroke. 2009;40(12):3828-3833; doi: 10.1161/STROKEAHA.109.561365.

5. Krakauer JW. Arm function after stroke: from physiology to recovery. Semin Neurol. 2005;25(4):384-395; doi: 10.1055/s-2005-923533.

6. Stoeckmann TM, Sullivan KJ, Scheidt RA. Elastic, viscous and mass load effects on poststroke muscle recruit- 
P. Vieira et al., Post-stroke reaching movements: augmented muscle activation

ment and co-contraction during reaching: a pilot study. Phys Ther. 2009;89(7):665-678; doi: 10.2522/ptj. 20080128.

7. Gowland C, deBruin H, Basmajian JV, Plews N, Burcea I. Agonist and antagonist activity during voluntary upper-limb movement in patients with stroke. Phys Ther. 1992;72(9):624-633; doi: 10.1093/ptj/72.9.624.

8. Wagner JM, Dromerick AW, Sahrmann SA, Lang CE. Upper extremity muscle activation during recovery of reaching in subjects with post-stroke hemiparesis. Clin Neurophysiol. 2007;118(1):164-176; doi: 10.1016/j. clinph.2006.09.022.

9. McCrea PH, Eng JJ, Hodgson AJ. Saturated muscle activation contributes to compensatory reaching strategies following stroke. J Neurophysiol. 2005;94(5):2999 3008; doi: 10.1152/jn.00732.2004.

10. Trombly CA. Deficits of reaching in subjects with left hemiparesis: a pilot study. Am J Occup Ther. 1992; 46(10):887-897; doi: 10.5014/ajot.46.10.887.

11. Hughes AM, Freeman CT, Burridge JH, Chappell PH, Lewin PL, Rogers E. Shoulder and elbow muscle activity during fully supported trajectory tracking in people who have had a stroke. J Electromyogr Kinesiol. 2010;20(3): 465-476; doi: 10.1016/j.jelekin.2009.08.001.

12. Massie CL, Malcolm MP, Greene DP, Browning RC. Kinematic motion analysis and muscle activation patterns of continuous reaching in survivors of stroke. J Mot Behav. 2012;44(3):213-222; doi: 10.1080/00222895. 2012.681321.

13. Castiello U. The neuroscience of grasping. Nat Rev Neurosci. 2005;6(9):726-736; doi: 10.1038/nrn1744.

14. Jeannerod M. The timing of natural prehension movements. J Mot Behav. 1984;16(3):235-254; doi: 10.1080/ 00222895.1984.10735319.

15. Cirstea MC, Levin MF. Compensatory strategies for reaching in stroke. Brain. 2000;123(Pt 5):940-953; doi: 10.1093/brain/123.5.940.

16. Avila MA, Romaguera F, Oliveira AB, Camargo PR, Salvini TF. Bilateral impairments of shoulder abduction in chronic hemiparesis: electromyographic patterns and isokinetic muscle performance. J Electromyogr Kinesiol. 2013;23(3):712-720; doi: 10.1016/j.jelekin.2012.12.001.

17. Krakauer JW, Carmichael ST, Corbett D, Wittenberg GF. Getting neurorehabilitation right: what can be learned from animal models? Neurorehabil Neural Repair. 2012;26(8):923-931; doi: 10.1177/154596831 2440745.

18. Raimundo KC, Silveira LS, Kishi MS, Fernandes LFRM, Souza LAPS. Kinematic and electromyographic analysis of the reaching in stroke patients [in Portuguese]. Fisioter Mov. 2011;24(1):87-97; doi: 10.1590/ S0103-51502011000100010.

19. Wu CY, Chen CL, Tang SF, Lin KC, Huang YY. Kinematic and clinical analyses of upper-extremity movements after constraint-induced movement therapy in patients with stroke: a randomized controlled trial.
Arch Phys Med Rehabil. 2007;88(8):964-970; doi: 10.1016/j.apmr.2007.05.012.

20. Thielman G, Kaminski T, Gentile AM. Rehabilitation of reaching after stroke: comparing 2 training protocols utilizing trunk restraint. Neurorehabil Neural Repair. 2008;22(6):697-705;doi:10.1177/1545968308315998.

21. Hermens HJ, Freriks B, Disselhorst-Klug C, Rau G. Development of recommendations for SEMG sensors and sensor placement procedures. J Electromyogr Kinesiol. 2000;10(5):361-374; doi: 10.1016/s1050-6411(00) 00027-4.

22. Lima FM, Abreu RS, Shimano GN, Souza LAPS, Fernandes LFRM. Methodological proposal for surface electrodes positioning on the wrist and fingers muscles through anthropometric measurements of forearm. Braz J Oral Sci. 2010;9(4):208.

23. Oliveira RA, Vieira PCS, Fernandes LFRM, Patrizzi LJ, Oliveira SF, Souza LAPS. Mental practice and mirror therapy associated with conventional physical therapy training on the hemiparetic upper limp in poststroke rehabilitation: a preliminary study. Top Stroke Rehabil. 2014;21(6):484-494; doi: 10.1310/tsr2106-484.

24. Hammond MC, Fitts SS, Kraft GH, Nutter PB, Trotter MJ, Robinson LM. Co-contraction in the hemiparetic forearm: quantitative EMG evaluation. Arch Phys Med Rehabil. 1988;69(5):348-351.

25. Chae J, Yang G, Park BK, Labatia I. Delay in initiation and termination of muscle contraction, motor impairment, and physical disability in upper limb hemiparesis. Muscle Nerve. 2002;25(4):568-575; doi: 10.1002/ mus.10061.

26. Chang SH, Francisco GE, Zhou P, Rymer WZ, Li S. Spasticity, weakness, force variability, and sustained spontaneous motor unit discharges of resting spasticparetic biceps brachii muscles in chronic stroke. Muscle Nerve. 2013;48(1):85-92; doi: 10.1002/mus.23699.

27. Tang A, Rymer WZ. Abnormal force-EMG relations in paretic limbs of hemiparetic human subjects. J Neurol Neurosurg Psychiatry. 1981;44(8):690-698; doi: 10.1136/jnnp.44.8.690.

28. Taketomi Y, Muraki T. Dynamometer testing of upper trapezius muscle strength. Bull Allied Med Sci Kobe. 1989;5:83-88.

29. Twitchell TE. The restoration of motor function following hemiplegia in man. Brain. 1951;74(4):443-480; doi: 10.1093/brain/74.4.443.

30. Boyd LA, Vidoni ED, Wessel BD. Motor learning after stroke: is skill acquisition a prerequisite for contralesional neuroplastic change? Neurosci Lett. 2010;482(1): 21-25; doi: 10.1016/j.neulet.2010.06.082. 